\title{
Mandenkan
}

MANDENIKAN Bulletin semestriel d'études linguistiques mandé

$52 \mid 2014$

Numéro 52

\section{Remarques à propos des « Propositions pour l'orthographe du bambara »}

Some remarks on the "Proposals for the orthography of Bamanankan"

НЕКОТОРЫЕ ЗАМЕЧАНИЯ К «ПРЕДЛОЖЕНИЯМ К ОРФОГРАФИИ ЯЗЫКА БАМАНА»

\section{Gérard Dumestre}

\section{OpenEdition}

Journals

Édition électronique

URL : https://journals.openedition.org/mandenkan/326

DOI : $10.4000 /$ mandenkan.326

ISSN : 2104-371X

\section{Éditeur}

Llacan UMR 8135 CNRS/Inalco

Édition imprimée

Date de publication : 1 décembre 2014

Pagination : $55-59$

ISSN : 0752-5443

\section{Référence électronique}

Gérard Dumestre, «Remarques à propos des « Propositions pour l'orthographe du bambara » », Mandenkan [En ligne], 52 | 2014, mis en ligne le 01 décembre 2014, consulté le 08 juillet 2021. URL http://journals.openedition.org/mandenkan/326 ; DOI : https://doi.org/10.4000/mandenkan.326

Ce document a été généré automatiquement le 8 juillet 2021.

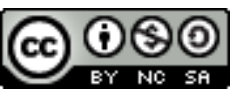

Les contenus de Mandenkan sont mis à disposition selon les termes de la Licence Creative Commons Attribution - Pas d'Utilisation Commerciale - Partage dans les Mêmes Conditions 4.0 International. 


\title{
Remarques à propos des «Propositions pour l'orthographe du bambara »
}

\author{
Some remarks on the "Proposals for the orthography of Bamanankan" \\ НЕКОТОРЫЕ ЗАМЕЧАНИЯ К «ПРЕДЛОЖЕНИЯМ К ОРФОГРАФИИ ЯЗЫКА БАМАНА»
}

\author{
Gérard Dumestre
}

\section{NOTE DE L'AUTEUR}

Cette recherche s'insère dans le programme Investissements d'Avenir géré par

l'Agence Nationale de la Recherche ANR-10-LABX-0083 (Labex EFL, Axe 6).

Sur la plupart des points du guide proposé par Konta et Vydrine dans ce numéro de Mandenkan, j'exprime mon accord, et remercie les auteurs de ces mises au point nécessaires qui j'espère entreront prochainement dans les règles officielles ainsi que dans la pratique de l'écriture du bambara. Je voudrais simplement ici faire quelques observations, soit pour les compléter, soit pour exprimer une réserve. J'ajouterai également quelques problèmes qui ne sont pas abordés.

1. La nasale palatale. D'abord écrite ny, elle est désormais écrite $n$. Cette dernière graphie entraîne de fréquentes fautes. Lorsqu'un scripteur a affaire à ce son, il doit se poser la question de l'étymologie du terme: si le terme est composé, ou plus fréquemment dérivé, il faut écrire ny (fảdenya 'rivalité'), et dans le cas contraire il faut écrire $\Omega$ (kóno 'noce'). La difficulté augmente dans le cas où certains termes ne sont pas immédiatement analysables comme des termes complexes. Dans le cas du verbe 'égaliser', de kán et -ya, l'écriture correcte kénye n'est pas évidente. On objectera que

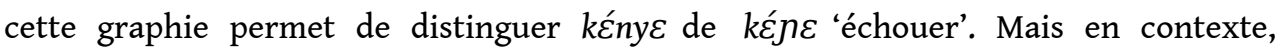
rarissimes seront les cas où apparaît une ambigüité entre les deux termes. Le choix dans tous les cas de ny est plus simple à la fois pour le lecteur et le scripteur, et permet d'éviter une lettre qui, à l'évidence, ne se trouvera pas sur les claviers ordinaires.... et 
qu'on écrira sans doute n'importe comment (gn par exemple dans les publicités actuellement placardées dans Bamako).

2 2. Les deux voyelles ouvertes $\varepsilon$ et 0 . Là encore, et pendant plusieurs années, on a écrit è et $o$. Le passage aux nouvelles lettres s'est opéré afin d'aligner les règles d'orthographe sur celles en cours en Côte d'Ivoire. L'avantage est mince, les publications ivoiriennes circulant aussi peu au Mali que celles du Mali en Côte d'Ivoire. La principale conséquence de ce changement, comme pour la nasale palatale, est que l'écriture « correcte » du bambara et réservée à ceux qui possèdent le clavier spécial, c'est-à-dire à très peu de personnes. Et cela explique aussi que dans les médias modernes (publicités, enseignes, blogs, etc) les voyelles ouvertes et fermées sont le plus souvent non distinguées. Pour une orthographe du bambara utilisable partout et par tous, ne vaudrait-il pas mieux revenir aux graphies ny, è et $\hat{o}$ ?

3 3. Le cas des termes finissant par consonne nasale + in / un est délicat. La possibilité d'une épenthèse nasale terminale (dans les cas de composition ou de dérivation par exemple) est l'un des critères pouvant déterminer si l'on doit ou non noter la nasalité de la voyelle: on entend systématiquement dùnunba et non du nuba, et donc il convient d'écrire dùnun; on entend à ní dén nàna et ní doit s'écrire sans nasale; on entend nìn (n)dén et le démonstratif doit s'écrire nìn. Un examen précis de chaque terme est indispensable.

4 4. Il conviendrait de faire un inventaire complet (les cas sont assez peu nombreux) des termes dont la forme courante est ClV et dont la voyelle disparue est « disputée » : par exemple dòloki et dùlıki (on trouve dans les textes les deux graphies), et d'en proposer une orthographe définitive.

5 5. Je suis en désaccord avec l'orthographe séparée des nombres comprenant bí : il me semble préférable de les écrire en une seule unité graphique : bísaba 'trente', bínaani 'quarante', bíduuru 'cinquante'... Tout d'abord parce que bí , contrairement à kغ̀me 'cent' n'est jamais utilisé seul, ensuite et surtout parce que lorsque le nombre est complexe, la lecture me semble en être facilitée : kème sàba ní bísaba ní dúuru 'trois cent trente cinq' plutôt que kème sàba ní bí sàba ní dúuru, ou wáa binaani 'deux cent mille (francs CFA)' plutôt que wáa bí náani. Même si ce n'est pas un argument décisif, remarquons qu'en français, en anglais, en espagnol ou en allemand, des formes comparables, et construites d'une manière assez analogue s'écrivent aussi en une seule unité : cinquante, fifty, cincuenta, fünfzig.

6. L'orthographe des tons. Observons tout d'abord que, depuis bientôt un demi-siècle, si l'on excepte le premier lexique bambara-français où il sont notés, les tons ne figurent dans aucun texte édité au Mali, qu'il s'agisse de livrets pédagogiques, de journaux, ou de textes littéraires publiés par des Maliens. Aucune publication linguistique malienne non plus ne comporte de textes où les tons seraient indiqués. Ajoutons (et c'est l'expérience de l'enseignement du bambara qui me l'a montré) qu'un texte sans tons mais correctement écrit se lit sans difficulté, et sans problème de compréhension dans l'immense majorité des cas. Il est d'ailleurs très intéressant de constater que la lecture d'un texte sans tons, par des non-locuteurs natifs ayant suivi un enseignement de la grammaire bambara, se fait sans grande faute d'intonation: la connaissance de la syntaxe, une écriture correcte des mots et groupes de mots (composition, dérivation, syntagmes...) ainsi qu'une ponctuation précise pallient l'absence des tons dans quasiment tous les cas. Il m'apparaît donc que la notation tonale doit d'une part être réservée à ceux qui s'intéressent à la langue comme objet d'étude et non au contenu des 
textes, et d'autre part figurer comme une possibilité supplémentaire d'écriture pour ce qu'on pourrait appeler une graphie de niveau supérieur. Dans le premier cas, toutes les orthographes tonales sont possibles, y compris les plus complètes et donc les plus complexes : notation des tons sur toutes les syllabes, sur les noms propres, indication des modulations, article tonal.... Dans le second, il me semble suffisant de s'en tenir à la simple notation des tons sur la première syllabe des mots, en dehors des noms propres, cette notation permettant simplement l'identification des mots de la phrase (et ne fournissant pas d'information sur la prononciation en contexte de chaque élément).

7 7. Le redoublement. Je souscris (quoi qu'avec une certaine réticence) à l'idée de séparer les termes des formes redoublées par un trait d'union: et donc sògosogo 'tousser' (forme non sécable), mais sògo-sogo 'percer à plusieurs reprises', ou pour les adverbes expressifs : kúlukulu 'complètement (finir)', mais cóyi-coyi 'très (rouge)'. Il faut exclure de cette règle les adverbes expressifs ou les verbes de redoublement partiel (par exemple les redoublés en $a$ : kólonkalan 'de grande taille' (de kòlon 'pilon'). Il faut également exclure de cette règle les quelques noms redoublés, dont parfois la forme longue est de sens assez différent de celui de la forme courte : c⿱亠̀े cE 'doyen d'âge', túlontulon 'amusement', déndennin 'poupée'.

8 8. A la liste des séquences qui doivent s'écrire en une seule unité, il faut au moins ajouter dóin 'l'un' (dans une série de deux).

9 9. Les distinctions entre les deux formes béka et bé kà , et téka et té kà sont sans doute correctes au plan linguistique (même si pour l'exemple donné par les auteurs : À kÉra

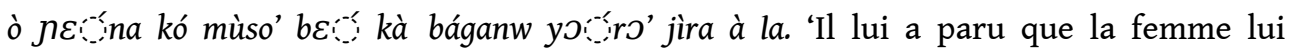
montrait (était en train de lui montrer) l'endroit où se trouvaient les bêtes', il me semble difficile de décider s'il s'agit d'un emploi du progressif ou du parfait inférentif. Quant à l'exemple À bésík táalen súgu' lá. '(Apparemment) il est parti au marché', il faudrait le vérifier auprès de plusieurs locuteurs, je n'ai pour ma part jamais entendu une telle construction. De toutes les façons, ces distinctions ne seront pas reconnues par les scripteurs, qui écriront indifféremment l'une ou l'autre. Il en ira de même pour les trois formes bé/té... lá (5.11) correspondant à trois constructions différentes.

10 10. Il sera nécessaire d'introduire dans les règles d'ortographe des éléments de ponctuation (point, points d'interrogation, d'exclamation, de suspension, deux points). L'usage de la virgule, essentielle pour une bonne lecture de la phrase, doit être précisé. Quant au point d'exclamation, il pourrait avantageusement être employé redoublé pour indiquer les répétitions au-delà du simple redoublement : à finna kíri! 'c'est très noir !' à finna kíri-kiri! 'c'est très très noir !', mais à finna kíri-kiri!! 'c'est très très très noir !' (pour à finna kíri-kiri-kiri-kiri (répétition 'à l'infini').

11 11. Le séquentiel. Je ne vois pas l'intérêt d'écrire les constructions séquentielles avec un trait d'union: une phrase comme ù fila fila nàna 'ils sont venus deux par deux' ne présente aucune ambiguïté de lecture; et dès lors qu'on utilise un trait d'union, il conviendrait d'écrire de la même manière lorsqu'il s'agir dune séquence de noms, donc entre les deux sòrosabu de la phrase: dije yé sòrosabu-sorosabu dàma yé 'le monde entier n'est fait que d'une suite de causes qui s'enchaînent', ce qui paraît inutilement lourd.

12 12. Les conglomérés. Choisir une graphie de ces formes en fonction des tons, dans la mesure où ces contructions sont relativement ouvertes (et donc qu'il est difficile d'en faire un recensement complet), me semble inadéquat, et propice à permettre toutes les fantaisies d'écriture. Il me semble préférable de proposer l'écriture avec traits d'union 
pour les formes les plus longues: nà-n-n-k'i-nyininka 'Acacia ataxacantha', ou á-ye-fali$b \varepsilon n$ 'vêtement de grande ampleur'.

Une remarque terminale : Si l'on examine les textes publiés en bambara, par exemple les articles des journaux, on remarque que les règles d'orthographe (graphie des lettres, césures entre les constructions, etc.) sont assez bien respectées, mais que les phrases sont souvent très difficiles à lire, pour trois raisons : 1) les espacements entre les mots sont insuffisants, et parfois quasi-absents; 2) de nombreuses coquilles apparaissent, pour cause d'absence d'une relecture soignée 3) les phrases sont le plus souvent traduites du français, et non réinterprétées et récrites dans la syntaxe propre au bambara, ce qui entraîne des lourdeurs et des incompréhensions.

\section{RÉSUMÉS}

Les propositions pour l'orthographe bambara de Mahamadou Konta et Valentin Vydrine sont excellentes dans la plupart des cas, cependant, quelques remarques peuvent être formulées. Par exemple, l'emploi des signes phonétiques $\mathrm{O}, \varepsilon, \mathrm{n}$ (là où on pourrait se passer des digraphes ou des lettres accentuées) ; l'écriture séparée des noms de dizaines (une écriture collée me semble préférable) ; l'écriture des marques du « parfait inférentiel »; l'utilisation des traits d'union. Il serait souhaitable de réglementer l'utilisation de la ponctuation.

Proposals for the Bambara orthography by Mahamadou Konta and Valentin Vydrin are excellent. However, there are certain points where some reservations can be expressed: the use of phonetic symbols $\mathrm{O}, \varepsilon, \mathrm{n}$ (digraphs and accented letters could be used instead: ò, è, ny); separate spelling of the names for tens (a writing as one solid word seems to be preferable: bisaba 30, biduuru 50, etc.); spelling of the "inferential perfect" marker; use of a hyphen. A reglementation of the ponctuation marks would be desirable.

ПРЕДЛОЖЕНИЯ ПО ОРФОГРАФИИ БАМАНА, СФОРМУЛИРОВАННЫЕ МАХАМАДУ КОНТА И ВАЛЕНТИНОМ ВЫДРИНЫМ, В ОСНОВНОМ ПРЕДСТАВЛЯЮТСЯ ВЕСЬМА УДАЧНЫМИ. ТЕМ НЕ МЕНЕЕ, ПО НЕКОТОРЫМ ПУНКТАМ СЛЕДУЕТ СДЕЛАТЬ ОГОВОРКИ. ЭТО КАСАЕТСЯ, В ЧАСТ-НОСТИ, СЛЕДУЮЩЕГО: ИСПОЛЬЗОВАНИЯ ФОНЕТИЧЕСКИХ СИМВОЛОВ, , $\varepsilon$, $\mathrm{n}$ (МОЖНО БЫЛО БЫ ОБОЙТИСЬ ДИГРАФАМИ И БУКВАМИ С АКЦЕНТАМИ: о̀, ’̀, ПУ); РАЗДЕЛЬНОГО НАПИСАНИЯ НАЗВАНИЙ ДЕСЯТКОВ (СЛИТНОЕ НАПИСАНИЕ ПРЕДСТАВЛЯЕТСЯ БОЛЕЕ ПРЕДПОЧТИТЕЛЬНЫМ: bisaba 30, biduuru 50, И Т.Д.); ПРАВОПИСАНИЕ ПОКАЗАТЕЛЯ «ИНФЕРЕНТИВНОГО ПЕРФЕКТА»; ИСПОЛЬЗОВАНИЕ ДЕФИСА. ПРЕДСТАВЛЯЕТСЯ ЖЕЛАТЕЛЬحНЫМ ТАКЖЕ РЕГЛА-МЕНТИРОВАТЬ ИСПОЛЬЗОВАНИЕ ЗНАКОВ ПУНКТУАЦИИ.

\section{INDEX}

Thèmes : bambara

motsclesru БАМАНА, ОРФОГРАФИЯ, ПУНКТУАЦИЯ

Keywords : Bambara, Orthography, Punctuation

Mots-clés : orthographe, ponctuation 
AUTEUR

GÉRARD DUMESTRE

LLACAN, INALCO, USPC

g.dumestre@wanadoo.fr 